Editor Científico: Prof. Dr. Martinho Isnard Avaliação: Double Blind Review pelo SEER/OJS Revisão: Gramatical, normativa e de formatação

\title{
QUEDA NO PREÇO DAS AÇÕES DOS TIMES DE FUTEBOL EUROPEU DURANTE A CRISE DO SUBPRIME
}

Doi 10.21902/emprad.v1i1.318

Erivaldo da Silva Carneiro Junior ${ }^{1}$ Daniel Reed Bergmann ${ }^{2}$

Recebido: $25 / 08 / 2016$

Aprovado: 20/10/2016

\section{RESUMO}

O futebol é um dos esportes mais praticados e profissionalizados no mundo, especialmente no mercado europeu. Diante dessa afirmativa é justificável a atenção que os times e clubes têm dispensado na gestão, procurando torná-la mais eficiente, atraindo a atenção de investidores para suas organizações de futebol (DANTAS et al., 2009). Nesse cenário de do investidor, vem a possibilidade da abertura de capital dos times e clubes, ao colocarem à venda as suas ações em bolsa de valores. Essa prática de obtenção de recursos tem sido bastante utilizada, especialmente por alguns times europeus, que acabou despertando interesse do mercado em criar índices para essa indústria, como é o caso do Bloomberg European Football Club Index Eurokick.

Palavras-chave: Ações; Futebol e Crise.

\footnotetext{
${ }^{1}$ Doutorando pela Universidade Nove de Julho - UNINOVE, São Paulo (Brasil)

E-mail: erivaldo carneiro@yahoo.com.br

${ }^{2}$ Doutor pela Universidade de São Paulo - USP, São Paulo (Brasil)

Professor pela Faculdade de Economia, Administração e Contabilidade - FEAC/USP, São Paulo

(Brasil). E-mail: danielrb@usp.br

${ }^{3}$ Doutor pela Universidade de São Paulo - USP, São Paulo (Brasil)

Professor pela Universidade Nove de Julho - UNINOVE, São Paulo (Brasil)

E-mail: juliocunha@yahoo.com
} 


\section{Introdução}

O futebol é um dos esportes mais praticados e profissionalizados no mundo, especialmente no mercado europeu. Diante dessa afirmativa é justificável a atenção que os times e clubes têm dispensado na gestão, procurando torná-la mais eficiente, atraindo a atenção de investidores para suas organizações de futebol (DANTAS et al., 2009). Nesse cenário de do investidor, vem a possibilidade da abertura de capital dos times e clubes, ao colocarem à venda as suas ações em bolsa de valores. Essa prática de obtenção de recursos tem sido bastante utilizada, especialmente por alguns times europeus, que acabou despertando interesse do mercado em criar índices para essa indústria, como é o caso do Bloomberg European Football Club Index - Eurokick.

De acordo o site Bloomberg, o European Football Club Index - Eurokick foi criado para fornecer medidas precisas da indústria do esporte, selecionadas com metodologias que refletem com precisão as características específicas do respectivo segmento. O Eurokick representa a amplitude e a profundidade da indústria do futebol europeu com todos os seus componentes sendo empresas listadas em bolsa, sendo composto por clubes de futebol da Europa e da Europa Oriental, cobrindo $100 \%$ do mercado alvo. Tendo em vista essa participação em bolsa de valores, os times de futebol, enquanto empresas de capital de aberto, estão sujeitos às variações, oscilações e riscos do mercado financeiro global e local.

Entre os anos de 2008 e 2009, o mundo assistiu à crise do subprime no mercado americano, que segundo MENDONÇA et al. (2011), foi desencadeada por conta dos maus resultados de 2007 e em março de 2008 de alguns banco e fundos de hipotecas. Diante do panorama apresentado ao Banco Central Americano - Fed, este se viu obrigado a intermediar o processo de venda do Bear Stearns ao JP Morgan Chase antecipando-se ao efeito que o risco sistêmico ligado a esta falência poderia originar. Em julho de 2008 as autoridades financeiras nos EUA prestaram assistência aos dois gigantes semi estatais do setor de hipotecas, Fannie Mae e Fredie Mac. Dois meses depois o governo assumiu o controle das duas empresas ao assumir que os níveis de endividamento destas instituições apresentavam um risco bem elevado para a estabilidade econômica. Tais atitudes do governo americano acabou gerando desconfiança dos investidores ao redor do mundo, instalando nos mercados globais a chamada crise do subprime americano.

De acordo SANVICENTE e MINARDI (1999), o custo de capital representa o retorno futuro esperado pelo investidor em uma determinada empresa. Esse retorno futuro vai depender do mercado em que a empresa está inserida e à medida que o risco do papel aumenta, a expectativa de retorno esperado pelo investidor também aumenta. Para calcular o retorno de títulos, o CAPM - Capital Asset Princig Model é o modelo utilizado para calcular o risco de mercado no qual a empresa está inserida e é medido pelo coeficiente Beta $\beta$.

Este relato técnico possui o objetivo de verificar o impacto da crise do subprime americano nos times de futebol europeu que possuem ações negociadas em bolsa de valores e integram o Índice Eurokick. Especificamente, procuramos descobrir se houve um aumento significativo do risco sistemático dos times europeus de futebol durante a crise internacional do subprime (2008/2009) através do cálculo do CAPM - Capital Asset Princig Model medido pelo índice Beta $(\beta)$, calculado para ramos específicos da economia. No caso em questão, trata-se da indústria do futebol europeu.

O presente relato técnico está apresentado em oito seções, a começar pela introdução. A seção 2 tem como foco o referencial teórico, a respeito dos eixos que o fundamentam: CAPM, e risco sistêmico. Na seção 3 o método da produção técnica é descrito. Em seguida, na seção 4, a contextualização da situação-problema é realizada, sendo caracterizada como a crise do 
subprime. Na seção 5 são delineados o tipo de intervenção e os mecanismos adotados que são através de um parecer técnico, com um diagnóstico de uma situação problema, ao passo que na seção 6 são apresentados os resultados obtidos e a análise realizada acerca da situação problema. Na seção 7, são feitas as considerações finais e as limitações do estudo. Por fim, na seção 8, são apresentadas as referências.

\section{Referencial Teórico}

De acordo ASSAF NETO, LIMA E DE ARAÚJO (2008), para calculo do custo de capital próprio o método utilizado é o CAPM (Capital Asset Pricing Model), um modelo que associa o risco e o retorno para determinado ativo e que pode ser calculado através da seguinte equação:

$$
\mathrm{k}_{\mathrm{j}}=\mathrm{R}_{\mathrm{f}}+\left[\beta_{\mathrm{j}} \mathrm{x}\left(\mathrm{k}_{\mathrm{m}}-\mathrm{R}_{\mathrm{f}}\right)\right]
$$

Onde,

$\mathrm{k}_{\mathrm{j}}=$ retorno exigido sobre o ativo $j$ (ou $\mathrm{k}_{\mathrm{e}}$, custo do capital próprio) Tesouro)

$\mathrm{R}_{\mathrm{f}}=$ taxa de retorno livre de risco (medida geralmente pelo retorno sobre o Título do

$\beta_{\mathrm{j}}=$ coeficiente beta do ativo $j$ (é um índice do grau de movimento do retorno do ativo $j$ em resposta à mudança no retorno do mercado)

O coeficiente beta é uma medida da sensibilidade da variação dos retornos de determinada ação em resposta a variações do retorno de alguma medida do mercado e é representado por como função da relação entre o retorno exigido sobre determinado ativo e o retorno exigido pelo mercado (ASSAF NETO, 2012).

SHINOHARA (2003), explica Este coeficiente beta para determinado ativo pode ser interpretado da seguinte maneira:

- se beta > 1,0: um coeficiente beta maior do que 1 significa que, quando a taxa de retorno do mercado move-se, seja para cima ou para baixo, a taxa de retorno exigida sobre determinado ativo tende a se mover no mesmo sentido, porém em maior magnitude.

- $\quad$ se beta $=1,0$ : um coeficiente beta igual a 1 significa que, quando a taxa de retorno do mercado varia, a taxa de retorno exigida sobre determinado ativo também varia no mesmo sentido na mesma proporção;

- se $1<$ beta $\leq 0$ : um coeficiente beta menor do que 1 e maior ou igual a 0 significa que, quando a taxa de retorno do mercado move-se, seja para cima ou para baixo, a taxa de retorno exigida sobre determinado ativo tende a se mover no mesmo sentido, porém em menor magnitude.

- se beta < 0: neste raro caso, um coeficiente beta menor do que zero significa que, quando a taxa de retorno de mercado move-se, seja para cima ou para baixo, a taxa de retorno exigida sobre determinado ativo tende a se mover no sentido contrário.

A principal aplicação do modelo CAPM é para determinar o custo de capital próprio de uma empresa, ou seja, o quanto os investidores estão dispostos a receber pelo capital investido na respectiva empresa. Conforme MARTELANC, PASIN e PEREIRA (2010), as quatro principais formas de aplicação do conceito de custo de capital nas decisões das empresas são:

- avaliação do desempenho empresarial: uma empresa agrega valor aos seus acionistas na exata medida em que o retorno trazida por suas ações excede o custo desses acionistas de capital; 
- avaliação dos investimentos: o nível de investimento na economia é condicionada pelo custo de capital para as empresas. Desta forma, a melhor alternativa de uso de recursos com o objetivo de maximizar a riqueza do seu proprietário. No entanto, o autor destaca como exemplo o uso do custo de capital na avaliação de viabilidade econômica de projetos de investimento da empresa, ou na avaliação de empresas para fusões e aquisições, reestruturações e de abertura ou fechamento do capital;

- avaliação da decisões de financiamento: o custo de capital é usado para apoiar as decisões de uma empresa de financiamento, ou de terceiros. O autor fornece exemplos como a seleção de alternativas de financiamento para o presente método de valor líquido, a determinação da viabilidade e do preço de lançamento das ações e a otimização da estrutura de capital; as operações da empresa: o custo de capital também é usado em decisões operacionais de gestão empresarial, tais como a fixação de metas para a realização de resultados futuros, as decisões de compras ou vendas, otimização de volumes de estoques e determinação do preço de mercado, com base no custo de capital da empresa.

Comparamos neste estudo os betas mensais gerados ao longo do tempo para o índice Eurokick. Escolhemos como carteira de mercado o índice S\&P 500, pois pretendemos avaliar o impacto da crise norte-americana do subprime em tal índice. Bonomo and Garcia (2001) mostram que o CAPM condicional pode ser expresso pela seguinte equação:

$E\left[r_{i}(i) \mid L_{i}\right\rceil=\beta_{i i} E\left[r_{w}(i) \mid i u_{t}\right\rceil$

Onde $\mathrm{r}_{\mathrm{i}}(\mathrm{t})$ é o retorno em excesso (retorno do Eurokick acima do T-Bond de 10 anos) durante o período $i$ da carteira; $\mathrm{r}_{\mathrm{M}}(\mathrm{t})$ é o retorno em excesso da carteira de mercado; e $\beta_{\text {it }}$ é dado por:

$$
\begin{aligned}
& \beta_{i t}=\frac{\operatorname{cov}\left[r_{1}(i), r_{N}(i) \mid n_{i}\right]}{k a r\left[r_{N} l i\right) n_{1}}
\end{aligned}
$$

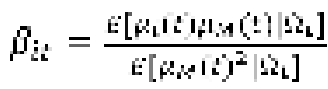

As equações que expressam $r_{i}(t)$ e $r_{M}(t)$ como modelos auto regressivos são dadas por:

$$
\begin{aligned}
& E\left[\mu_{1}(i) \mu_{i i}(i) \mid \Omega_{i}\right]=\delta_{0 i}+\sum_{i=:}^{k_{i}} \delta_{i i} \mu_{i}(i-j) \mu_{i n}(l-i)
\end{aligned}
$$

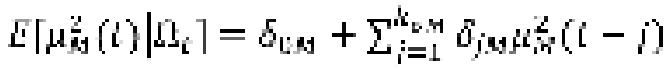

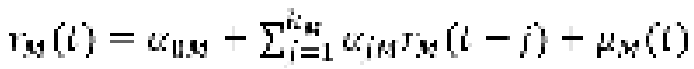

$$
\begin{aligned}
& r_{i}(l)=a_{i j i}+\sum_{j=1}^{k_{i}} a_{j i} r_{i}(l-\hat{j})+\mu_{1}(i)
\end{aligned}
$$

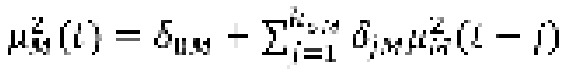

Neste CAPM, toda informação disponível durante o tempo $t$ tendem a ser capturadas pelo modelo auto regressivo acima. O beta será determinado pela seguinte equação através do software E-Views 6.0.

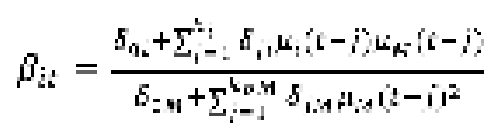

O risco total de um ativo pode ser avaliado em duas partes: sistemático e diversificável. A parcela do risco sistemático é determinada por fatores conjunturais e de mercado que atingem todas as empresas. Esse risco permanece na carteira independe da diversificação, sendo relevante para todo investidor. O risco diversificável é aquele que pode ser eliminado pela diversificação. Esse risco não deve preocupar o investidor diversificado, sendo 
identificado apenas no contexto específico da empresa. Um investidor diversificado deve exigir remuneração apenas do risco sistemático incorrido (ASSAF NETO, LIMA e DE ARAÚJO, 2008).

Em conformidade com FÁVERO, (2001, p. 75) "gerenciar riscos torna-se fundamental para os dirigentes das organizações, pois está relacionado com o potencial de agregação de valor, dependendo das características de cada dimensão de risco e das expectativas de retorno". Ainda de acordo FÁVERO, (2001, p. 75), outra questão a ser observada refere-se à "obtenção e entendimento dos objetivos estratégicos e operacionais da instituição, incluindo fatores críticos de sucesso, ameaças e oportunidades relacionadas".

Para estudar o risco sistêmico é necessário entender o grau de incerteza existente no sistema resultante de variações no nível de risco do crédito, da taxa de juros e do câmbio. A medição é realizada pelo impacto da variação do risco sobre o patrimônio líquido do sistema. Quanto maior a perda não-esperada potencial em relação ao patrimônio líquido, maior é o risco sistêmico. A crise é configurada no estresse do risco sistêmico, quando todos perdem a credibilidade na continuidade do sistema (CAPELLETTO, MARTINS E CORRAR 2008).

\section{Metodologia da Produção Técnica}

Trata-se de um estudo de cunho descritivo-exploratório, na medida em que busca descrever e compreender as características de uma determinada situação ou fenômeno. $\mathrm{O}$ propósito do estudo é observar fenômenos, procurando descrevê-los, classificá-los e interpretá-los. Possui também viés exploratório por se tratar de estudo de um ambiente específico, não estudado anteriormente e não passível de generalização. (BEUREN, 2008)

Este trabalho é considerado um estudo quantitativo, baseado em técnicas econométricas para o cálculo do beta bem como para a medição do risco de mercado pelo risco de mercado do Beta do CAPM. O programa estatístico que foram rodadas as informações foi o Gretl para Windows. A amostra analisada foi formada pelos times de futebol europeu que compõe o índice European Football Club Index - Eurokick, e possuíam suas informações no site da Bloomberg, que foram coletadas no dia 24/07/2013, totalizando 28 times, conforme tabela 1:

Tabela 1 - Composição dos times de futebol europeu participantes do índice Eurokick

\begin{tabular}{|l|l|}
\hline \multicolumn{1}{|c|}{ Time } & País \\
\hline Aalborg Boldspilklub A/S & Dinamarca \\
\hline Aarhus Elite A/S & Dinamarca \\
\hline AFC Ajax NV & Holanda \\
\hline AIK Fotboll AB & Suécia \\
\hline Arsenal Holdings PLC & Reino Unido \\
\hline AS Roma SpA & Itália \\
\hline Besiktas Futbol Yatirimlari Sanayi ve Ti & Turquia \\
\hline Borussia Dortmund GmbH \& Co KGaA & Alemanha \\
\hline Brondbyernes IF Fodbold A/S & Dinamarca \\
\hline Celtic PLC & Reino Unido \\
\hline EfB Elite A/S & Dinamarca \\
\hline FCM Holding A/S & Dinamarca \\
\hline Financiere Croissance Investis & França \\
\hline Fodboldalliancen AC Horsens AS & Dinamarca \\
\hline Futebol Clube Do Porto & Portugal \\
\hline GKS GieKSa Katowice AS & Polônia \\
\hline Juventus Football Club SpA & Itália \\
\hline
\end{tabular}




\begin{tabular}{|l|l|}
\hline OL Groupe & França \\
\hline Parken Sport \& Entertainment A/S & Dinamarca \\
\hline Rangers International Football Club PLC & Reino Unido \\
\hline Ruch Chorzow AS & Polônia \\
\hline Sileks Kratovo & Macedônia \\
\hline Silkeborg IF Invest A/S & Dinamarca \\
\hline Societa Sportiva Lazio SpA & Itália \\
\hline Sport Lisboa e Benfica-Futebol SAD & Portugal \\
\hline Sporting Clube de Braga & Portugal \\
\hline Sporting Clube De Portugal - Futebol SAD & Portugal \\
\hline Teteks Tetovo & Macedônia \\
\hline
\end{tabular}

Fonte: Bloomberg, 2013.

\section{Situação Problema}

Em agosto de 2007, foi detectada uma alta taxa de inadimplência no segmento que engloba pessoas com histórico de oferecerem menos garantias de pagamento, trata-se de clientes conhecidos como subprime. Por ter riscos maiores, os juros também são maiores, tornando-os mais atrativos para os gestores de fundos e bancos, que buscam retornos superiores (FERREIRA E PENIN, 2008).

De acordo LUZ SILVA, SANTOS e MOREIRA, (2011) não existe muita concordância entre analistas sobre o que gerou a crise financeira internacional. Muitos aceitam que ela foi causada pela crescente inadimplência dos empréstimos das hipotecas do mercado imobiliário norte-americano, sendo estendida aos mercados mundiais, uma vez que grande parte dessas hipotecas foi securitizada e distribuída a investidores e bancos de investimentos internacionais.

Diante do exposto, a situação problema do presente relato técnico é verificar o impacto da crise do subprime norte-americano (2008-2009) no preço das ações dos clubes de futebol europeu com ações listadas em bolsa de valores.

\section{Tipo de Intervenção e Mecanismos Adotados}

O risco capturado ao longo da crise norte-americana do subprime será determinado pelo CAPM condicional proposto por Bonomo e Garcia (2001). Este modelo possui a vantagem de capturar a evolução do risco sistêmico ao longo do tempo para o índice Eurokick e, assim, verificarmos o impacto da crise norte-americana na maiores dos times em destaque na Tabela 1.

\section{Resultados Obtidos e Análise}

A tabela 2 apresenta o peso de cada uma das ações no European Football Club IndexEurokick e seu respectivo ticker. 
Tabela 2 - Peso no índice Eurokick dos times de futebol

\begin{tabular}{|l|l|l|}
\hline Ticker da Ação & \multicolumn{1}{|c|}{ Time } & Peso no Índice \% \\
\hline AAB DC Equity & Aalborg Boldspilklub A/S & 0,176473726 \\
\hline ELITEB DC Equity & Aarhus Elite A/S & 0,301099947 \\
\hline AJAX NA Equity & AFC Ajax NV & 6,178756042 \\
\hline AIKB NG Equity & AIK Fotboll AB & 0,169512893 \\
\hline AFC PZ Equity & Arsenal Holdings PLC & 51,58868496 \\
\hline ASR IM Equity & AS Roma SpA & 2,844865396 \\
\hline BJKAS TI Equity & Besiktas Futbol Yatirimlari Sanayi ve Ti & 6,241832261 \\
\hline BVB GY Equity & Borussia Dortmund GmbH \& Co KGaA & 8,719442454 \\
\hline BIFB DC Equity & Brondbyernes IF Fodbold A/S & 0,709927047 \\
\hline CCP LN Equity & Celtic PLC & 2,760011248 \\
\hline EFBELITE G4 Equity & EfB Elite A/S & 0,033715088 \\
\hline FCMH G4 Equity & FCM Holding A/S & 0,056932533 \\
\hline MLFCI FP Equity & Financiere Croissance Investis & 0,028829744 \\
\hline ACHO G4 Equity & Fodboldalliancen AC Horsens AS & 0,018890802 \\
\hline FCP PL Equity & Futebol Clube Do Porto & 0,206060335 \\
\hline GKS PW Equity & GKS GieKSa Katowice SA & 0,054689248 \\
\hline JUVE IM Equity & Juventus Football Club SpA & 9,067854965 \\
\hline OLG FP Equity & OL Groupe & 1,267238724 \\
\hline PARKEN DC Equity & Parken Sport \& Entertainment A/S & 4,911472083 \\
\hline RFC LN Equity & Rangers International Football Club PLC & 1,470192207 \\
\hline RCW PW Equity & Ruch Chorzow SA & 0,083641615 \\
\hline SIL MS Equity & Sileks Kratovo & 0,028465266 \\
\hline SIFB DC Equity & Silkeborg IF Invest A/S & 0,339847048 \\
\hline SSL IM Equity & Societa Sportiva Lazio SpA & 1,278270232 \\
\hline SLBEN PL Equity & Sport Lisboa e Benfica-Futebol SAD & 0,716174121 \\
\hline SCB PL Equity & Sporting Clube de Braga & 0,025643063 \\
\hline SCP PL Equity & Sporting Clube De Portugal - Futebol SAD & 0,589332558 \\
\hline TETE MS Equity & Teteks Tetovo & 0,132144391 \\
\hline
\end{tabular}

Fonte: Elaborado pelos autores

O Arsenal é o time que apresenta o maior peso na composição do índice, com 51,59\%; seguido do Juventus, com 9,07 \%; acompanhado do Borussia Dortmund, com 8,72\%.

Diante dos dados obtidos no provedor da Bloomberg observou-se que durante a crise do subprime americano o retorno do índice dos times de futebol europeu que compõem o European Football Club Index - Eurokick obtiveram uma queda expressiva. 
Figura 1 - Evolução do índice Eurokick ao longo do período de 2000-2012

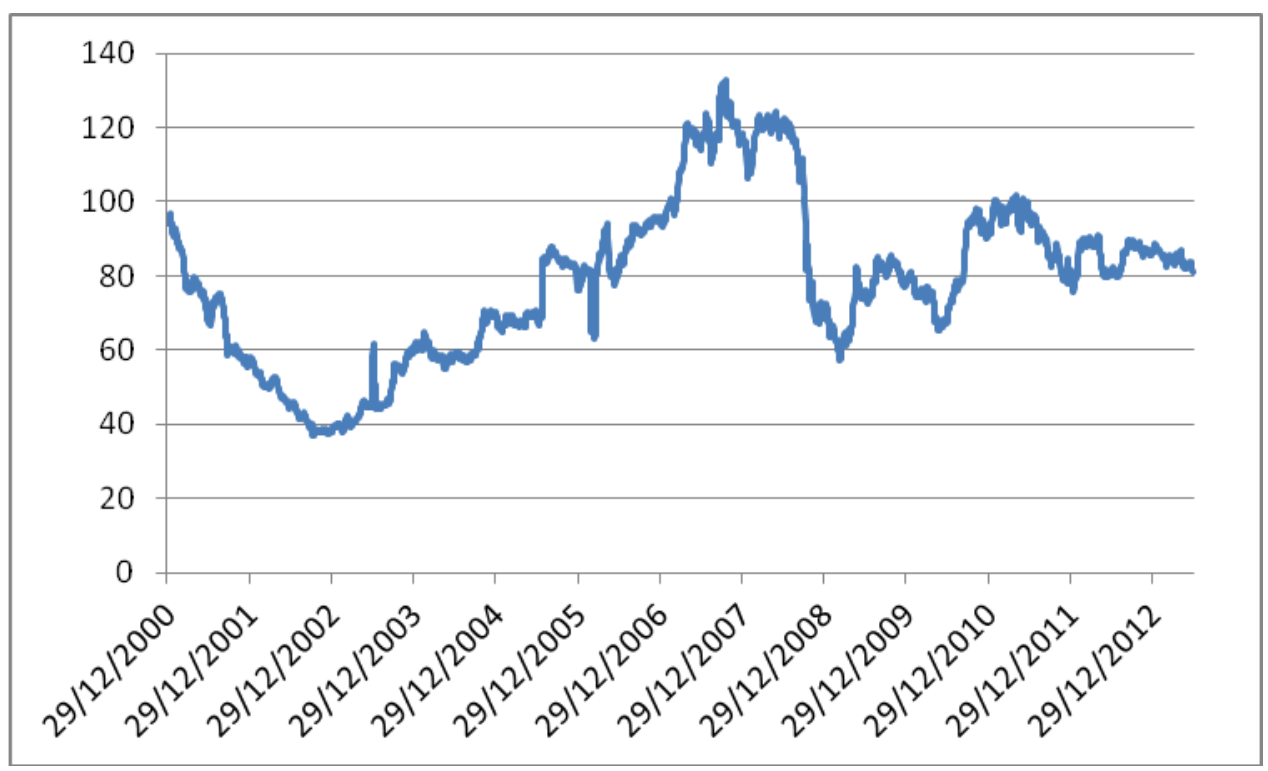

Fonte: Elaborado pelos autores

O gráfico que demonstra a evolução do risco sistemático do índice do Eurokick para o período de 2005 até 2013 é dado por:

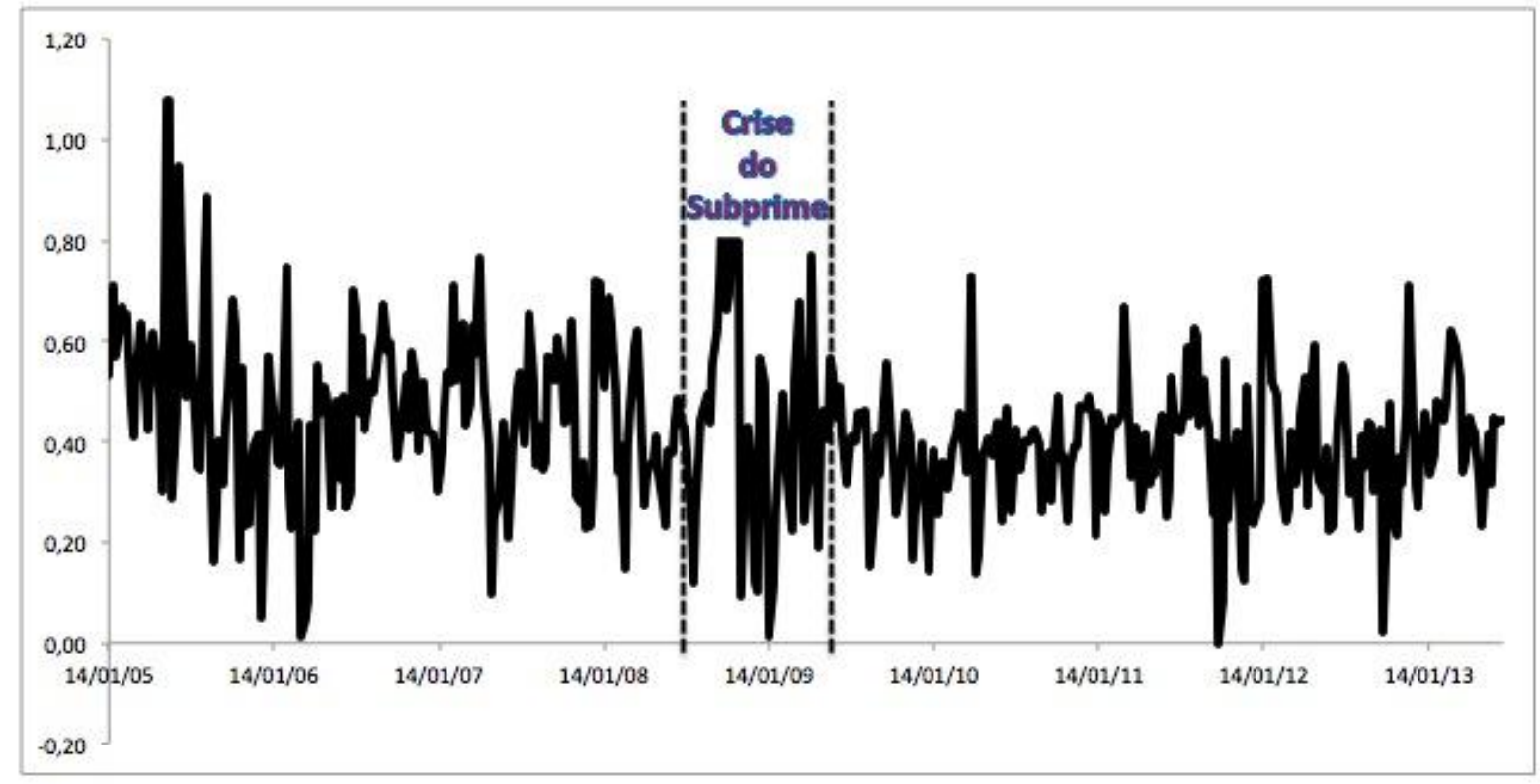

Realizou-se o teste de normalidade de Jarque-Bera para verificar se os betas acima seguem uma normal ao nível de 5\%. O valor-p do teste foi de 0,3292 e, portanto, não podemos rejeitar a hipótese nula de que os betas do índice Eurokick sigam uma normal. A tabela abaixo realiza o teste-F para verificar se a volatilidade do beta ao longo do tempo é maior do que no período anterior ( 24 meses que antecederam a crise). Os resultados obtidos são: 
Tabela 3 - Resultados obtidos para avaliar o impacto da crise do subprime no índice Eurokick

\begin{tabular}{|c|c|}
\hline Período & Variância dos betas \\
\hline$(1)-24$ meses anteriores a crise & 0,14 \\
\hline$(2)-$ período da crise do subprime $2008 / 2009$ & 0,31 \\
\hline$(2)-(1)$ & $0,17 * * *$ \\
\hline
\end{tabular}

Fonte: Elaborado pelos autores. $* * * 1 \%$, ** 5\% e * 10\% de nível de significância. Realizou-se o teste-F de variâncias para verificar se a volatilidade dos betas do índice do Eurokick sofreu um aumento significativo em relação aos 24 meses antecedentes a crise do subprime

Os resultados apontam para um aumento significativo do risco sistêmico nos times de futebol europeu durante a crise do subprime ao nível de 5\%. Assim, apesar dos times europeus não se envolverem operacionalmente com o mecanismo de derivativos de crédito no financiamento imobiliário norte-americano, tal crise afetou sobremaneira o preço das ações destas empresas. O beta médio durante todo período estudado foi de 0,43 , implicando que os times são, na média, investimentos conservadores para os investidores de mercado, pois se o S\&P 500 cair $1 \%$, tais times poderão ter um declínio nos retornos de $-0,43 \%$.

\section{Conclusão}

Estes resultados podem ser utilizados pelos investidores e/ou gestores dos clubes de futebol pois permitem um melhor entendimento das mudanças nos preços das ações do mercado de clubes futebol e seus determinantes de mercado, especialmente em períodos de crise sistêmica. Além disso, é importante para os gestores de clubes compreender o impacto das crises na volatilidade dos valores mobiliários bem como sua influência. Portanto, pelos resultados obtidos acima, um investidor que pretenda diversificar seus investimentos com empresas de times de futebol europeu na intenção de hedge, deve comparar a volatilidade do beta durante a crise ou períodos de alta volatilidade com outros setores da economia (e.g. utilities).

\section{Referências}

ASSAF NETO, A. et al. Uma proposta metodológica para o cálculo do custo de capital no Brasil. Revista de Administração da Universidade de São Paulo vol. 43.n. 1. p.72-83. 2008. Disponível em : < www.rausp.usp.br/download.asp?file=v4301072.pdf $>$. Acesso em: 06 jun. 2013.

ASSAF NETO, A. Finanças Corporativas e Valor. São Paulo: Atlas, 2003.

BEUREN, I. M. (Org. e col.). Como elaborar trabalhos monográficos em Contabilidade: teoria e prática. 3. ed., $2^{\mathrm{a}}$ reimp. São Paulo: Atlas, 2008.

BONOMO, M; GARCIA, R. Tests of conditional asset pricing models in the Brazilian stock Market. Journal of International Money and Finance, Vol. 12, p. 71-90, 2001.

BIANCOLINO, C. A. et al. Protocolo para elaboração de relatos de produção técnica doi: 10.5585/gep. v3i2. 121. Revista de Gestão e Projetos-e vol. 3 n.2, p. 294-307. 2012. 
CAPELLETTO, L. R.; MARTINS, E.; CORRAR, L. J. Mensuração do Risco Sistêmico no Setor Bancário com Variáveis Contábeis e Econômicas (No. 169). 2008. Disponível em: < www.bcb.gov.br/pec/wps/port/wps169.pdf >. Acesso em 09 jul. 2013.

DANTAS, M. G. S. et al.. O comportamento do preço das ações de clubes mediante a variação de aspectos contábeis: o estudo de caso do Juventus F.C. - Itália. Revista Ambiente Contábil, Natal-RN v. 1, n. 2, $2009 . \quad$ Disponível em: <http://ccsa.ufrn.br/ojs/index.php/ambiente/article/view/105/95>. Acesso em: 01 jul. 2013.

FÁVERO, A. E. Segurança em ambientes de comércio eletrônico. In: SILVA JUNIOR, J. B. Auditoria em ambiente de Internet. São Paulo: Atlas, 2001.

FERREIRA,T.T; PENIN, G. Evolução recente da crise do subprime: indicadores, reações $e$ perspectivas. Informações Fipe, maio de 2008. Disponível em: <http://www.fipe.org.br/publicacoes/downloads/bif/2008/5_16-20pen.pdf>. Acesso em 01 jul. 2013.

LUZ SILVA, R. M.; SANTOS, T. L.; MOREIRA, F. N. Crise do subprime: o papel da governança corporativa na volatilidade dos retornos. In: VIII ENCONTRO NORTE-RIOGRANDENSE DE CIÊNCIAS CONTÁBEIS, 2011, Natal. Anais Eletrônicos... Natal: CRC, 2011. Acesso em: < www.socialiris.org/antigo/imagem/boletim/arq4f8dc8b99ce7d.pdf>. Acesso em 15 jul. 2013.

MARTELANC, R.; PASIN, R.; PEREIRA, F. Avaliação de Empresas, Um guia para fusões e aquisições e private equity, $1^{\mathrm{a}}$ ed. São Paulo: Pearson. 2010.

MENDONCA, H. F.; GALVAO, D. J. C.; LOURES, R. F. V. Regulação e transparência: evidências a partir da crise do subprime. Econ. Apl. [online]. vol.15, n.1, pp. 23-44. 2011 Disponível em: 〈http://dx.doi.org/10.1590/S1413-80502011000100002〉. Acesso em 16 jun. 2013

SANVICENTE, A. Z.; MINARDI, A. M. A. F. Problemas de Estimação do Custo de Capital no Brasil. São Paulo: IBMEC, Relatório de Pesquisa. 1999.

SHINOHARA, D. Y. Estrutura de Capital e sua Relevância para a Organização. In: SEMINÁRIOS EM ADMINISTRAÇÃO, 2003, São Paulo. Anais Eletrônicos... São Paulo: USP, 2003. Disponível em: <http://www.ead.fea.usp.br/Semead/6semead/finan\%E7as/016Fin\%20Estrutura\%20do\%20Capital.doc.> Acesso em: 04 jul. 2013. 